\title{
Importance of polyethylene thickness in total shoulder arthroplasty: A finite element analysis
}

\author{
Alexandre Terrier ${ }^{\text {a,* }}$, Vittoria Brighenti ${ }^{a}$, Dominique P. Pioletti ${ }^{a}$, Alain Farron ${ }^{\mathrm{b}}$ \\ a Laboratory of Biomechanical Orthopedics, Ecole Polytechnique Fédérale de Lausanne, Station 19, 1015 Lausanne, Switzerland \\ b Service of Orthopaedics and Traumatology, University Hospital Center and University of Lausanne, Rue du Bugnon 46, 1011 Lausanne, Switzerland
}

\section{A R T I C L E I N F O}

\section{Article history:}

Received 11 October 2011

Accepted 6 December 2011

\section{Keywords:}

Total shoulder arthroplasty

Glenoid component

Polyethylene

Biomechanics

Finite element analysis

\begin{abstract}
A B S T R A C T
Background: Articular surfaces reconstruction is essential in total shoulder arthroplasty. Because of the limited glenoid bone support, thin glenoid component could improve anatomical reconstruction, but adverse mechanical effects might appear.

Methods: With a numerical musculoskeletal shoulder model, we analysed and compared three values of thickness of a typical all-polyethylene glenoid component: 2, 4 (reference) and $6 \mathrm{~mm}$. A loaded movement of abduction in the scapular plane was simulated. We evaluated the humeral head translation, the muscle moment arms, the joint force, the articular contact pattern, and the polyethylene and cement stress. Findings

Decreasing polyethylene thickness from 6 to $2 \mathrm{~mm}$ slightly increased humeral head translation and muscle moment arms. This induced a small decreased of the joint reaction force, but important increase of stress within the polyethylene and the cement mantel.

Interpretation
\end{abstract}

The reference thickness of $4 \mathrm{~mm}$ seems a good compromise to avoid stress concentration and joint stuffing. (c) 2011 Elsevier Ltd. All rights reserved.

\section{Introduction}

Although anatomical total shoulder arthroplasty is a satisfactory treatment for glenohumeral arthritis (Bohsali et al., 2006), there are still open questions to improve the design of the prosthesis or the surgical technique, and eventually increase the implant survival (Strauss et al., 2009). The thickness of all-polyethylene glenoid components is one of the design parameters that could be modified to reduce the failure rate of anatomical total shoulder arthroplasty.

The general idea of anatomical total shoulder arthroplasty is to reconstruct as much as possible the anatomical articular surfaces. In most of the cases, this objective can be achieved for the humeral side, thanks to the third generation modular prostheses. However, for the glenoid side, because of the constraint to keep as much as possible the subcondral bone support, the articular surface is actually displaced laterally by a few millimetres (Rockwood, 2009; Terrier et al., 2009a, 2009b). The thickness of current glenoid components vary from 3 to more than $15 \mathrm{~mm}$ (Rockwood, 2009). Thicker glenoid can reduce polyethylene stress and wear, but can induce joint stuffing, reduced mobility and instability (Harryman et al., 1995; Rockwood, 2009).

\footnotetext{
* Corresponding author at: Laboratory of Biomechanical Orthopedics, Ecole Polytechnique Fédérale de Lausanne, Station 19, 1015 Lausanne, Switzerland. E-mail address: alexandre.terrier@epfl.ch (A. Terrier).
}

For hip and knee arthroplasty, several studies have reported that the thickness of the polyethylene component is an important factor regarding its stress level and damage (Bartel et al., 1986; Oonishi et al., 1998). For the shoulder joint, the optimal thickness of the glenoid component has been only evaluated in two finite element analyses, which actually predict different effect of glenoid component thickness (Hopkins et al., 2007a, 2007b; Swieszkowski et al., 2003). None of these two studies focused on the thickness question. The first study was a mixed algebraic-numerical analysis of the contact stress at the glenohumeral surfaces (Swieszkowski et al., 2003). The authors showed that increasing the polyethylene thickness from 4 to $7 \mathrm{~mm}$ reduced the contact stress by about $25 \%$. The second study focused on polyethylene wear prediction as a function of various design parameters (Hopkins et al., 2007a, 2007b). In this study, glenoid components with 2, 6 and $10 \mathrm{~mm}$ of polyethylene thickness were evaluated. The peak contact pressure was nearly unaffected by the change of glenoid thickness, but the cement peak tensile stress was nearly 3-fold lower, as thickness increased from 2 to $10 \mathrm{~mm}$.

Thus, the effect of the thickness of the polyethylene component used in total shoulder arthroplasty seems still unclear. It is assumed that thinning the implant could improve the anatomical reconstruction of the articular surface, but we do not know the potential advantage or the drawback. Therefore, the goal of this study is to evaluate the biomechanical consequences of the thickness of the polyethylene glenoid component used in anatomical total shoulder arthroplasty. Starting from a typical existing glenoid component with a thickness 
of $4 \mathrm{~mm}$, we used a numerical model of the glenohumeral joint to evaluate two alternative thicknesses of $2 \mathrm{~mm}$ and $6 \mathrm{~mm}$.

\section{Methods}

A 3D numerical musculoskeletal model of the glenohumeral joint was used for this study (Fig. 1). This model is composed of the scapula, the humerus, the middle deltoid (MD), the anterior deltoid $(A D)$, the posterior deltoid (PD), the supraspinatus (SS), the subscapularis (SC), and the infraspinatus combined with the teres minor (IS). This model was built from CT data and anatomic dissection of a cadaveric shoulder without pathology. Around the humeral head, the MD, SS, SC and IS were modelled by rubber-like soft structure embedded with stiff longitudinal fibres. This allowed for the wrapping of these muscles around the humeral head, providing their stabilising effect. The AD and PD were modelled by stiff cables. All muscles were also given an active part, which was contracted with a specific force. This muscular force was automatically calculated by a feedback algorithm, which assumed fixed force ratios estimated from EMG measurements (Kronberg et al., 1990). During an imposed motion of the arm, this feedback algorithm is continuously predicting the muscular forces that solved the mechanical equilibrium, the contact stability and the muscle ratio constrain. The stabilising effect of the muscles is coupled with the glenohumeral contact to allow the natural translation of the humeral head within the glenoid fossa. A quasistatic movement of arm elevation in the plane of the scapula was simulated, from neutral elevation to $150^{\circ}$ of abduction, at full extension of the elbow. The arm weight was $37.5 \mathrm{~N}$ and $10 \mathrm{~N}$ was added in the hand. A scapulo-humeral rhythm of 2:1 was assumed to replicate the motion of the scapula and the correct alignment of the muscle action relative to the arm weight. Detail of this numerical musculoskeletal model and its validation can be found in technical (Terrier et al., 2007; Terrier et al., 2008b) and clinical application papers (Terrier et al., 2008a; Terrier et al., 2009a, 2009b; Terrier et al., 2010).

For the reference glenoid thickness of $4 \mathrm{~mm}$, we used the Aequalis anatomic prosthesis (Tornier Inc., Edina, MN, USA). The prosthesis was inserted into the numerical model according to manufacturer recommendations. The articular surfaces of each component were spherical. The diameter of the humeral head component was $48 \mathrm{~mm}$, which best match the humeral head radius. The radius of curvature of the glenoid component was $30 \mathrm{~mm}$, in order to follow the recommendation of a $6 \mathrm{~mm}$ radial mismatch between the humeral and glenoid surface. The humeral head component was optimally positioned to best fit the intact humeral head. The glenoid component was also positioned to best fit the glenoid articular surface, with a minimum resection of the glenoid subchondral bone and an optimal bone support. After the optimal virtual positioning of the components, the scapula and humerus were virtually cut to allow the final

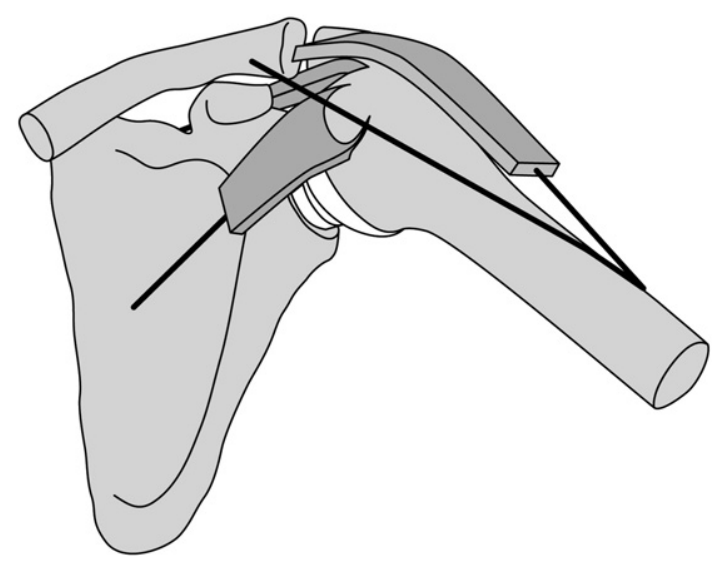

Fig. 1. Schematic illustration of the numeric musculoskeletal model used in this study. insertion of the two components. The virtual reaming, cementing, and positioning of the implants were performed with the standard modelling tools of the computer-aided design software SolidWorks (Dassault Systèmes, SolidWorks, Concord, MA, USA). The positioning of the glenoid and humeral components was assessed visually by a senior orthopaedic surgeon, using superposition of CT images with the positioned implants. A uniform layer of $0.5 \mathrm{~mm}$ of cement was placed between the host bone and the implants.

The polyethylene and cement were considered homogeneous and elastic (polyethylene: $\mathrm{E}=500 \mathrm{MPa}, \nu=0.4$; cement: $\mathrm{E}=2000 \mathrm{MPa}$, $\nu=0.3$ ). The local mechanical properties of the glenoid bone were obtained from the same set of CT images, using the relationship between Hounsfield number and elastic modulus (Carter and Hayes, 1977; Mansat et al., 1998; Rho et al., 1995). The articular contact was assumed frictionless. The two interfaces between the polyethylene, the cement and the bone were fully bounded. The finite element analysis was achieved with Abaqus 6.8 (www.simulia.com). Linear hexahedral elements were used for the polyethylene glenoid component and the cement mantel, while quadratic modified tetrahedral elements (C3D10M) were used for the glenoid bone. The metallic humeral component was modelled by a rigid analytic surface.

Two alternatives were compared to the reference glenoid thickness of $4 \mathrm{~mm}$ : a thinner $(2 \mathrm{~mm})$ and a thicker $(6 \mathrm{~mm})$ glenoid component. Using the original reference glenoid thickness of $4 \mathrm{~mm}$, we changed the thickness of the component using the above CAD software. The bone cut and positioning of the prosthetic components were the same for each case. For the three tested configurations, we evaluated the muscle moment arms, the muscle forces, the glenohumeral reaction force, the humeral head translation, the articular contact pressure, the von Mises stress within the polyethylene, and the maximum principal stress within the cement mantel. At the polyethylene-cement and cement-bone interface the shear stress was calculated. Within bone, strain was evaluated. The volume of polyethylene, cement and bone above a critical value was evaluated. The relative fraction of interfacial shear stress above a critical value was also evaluated. Kinematics, muscle moment arms and forces were evaluate during the entire abduction movement, but stress was evaluated only every $30^{\circ}$ of abduction.

\section{Results}

For sake of clarity, we present the effect of the glenoid thickness as an increasing value, although the $4 \mathrm{~mm}$ case is the reference value of the existing glenoid component.

\subsection{Kinematics}

During abduction, the translation of the humeral head centre relative to the glenoid followed an up and down movement. The humeral head centre migrated superiorly by about $3 \mathrm{~mm}$ during the first $30^{\circ}$ of abduction and then went down by $2 \mathrm{~mm}$ from 30 to $150^{\circ}$ of abduction. The increase of polyethylene thickness reduced the maximal upward migration of the humeral head by $0.2 \mathrm{~mm}$ (Fig. 2).

\subsection{Moment arms}

For the simulated movement, the moment arms of the 6 scapulohumeral muscles decreased as the thickness of the glenoid component increased. The maximal change of muscle moment arm was $2 \mathrm{~mm}$. In overall, this effect increased with abduction angle.

\subsection{Forces}

Muscle forces increased as the thickness of the glenoid component increased. The maximal change of muscle force was $4 \mathrm{~N}$. The increase of muscular forces induced an increase of the joint reaction force. The 


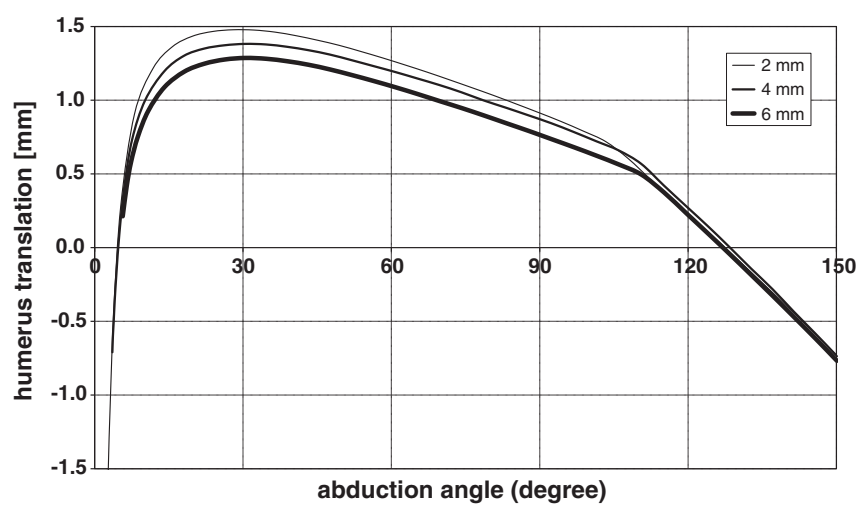

Fig. 2. Inferior-superior translation of the humeral head centre relative to the centre of glenoid fossa during abduction.

join reaction force was maximal at about $90^{\circ}$ of abduction. It reached $955 \mathrm{~N}, 971 \mathrm{~N}$ and $983 \mathrm{~N}$ for $2 \mathrm{~mm}, 4 \mathrm{~mm}$ and $6 \mathrm{~mm}$ of polyethylene thickness. This corresponded to an increase of $7 \mathrm{~N}$ per millimetre of thickness increase.

\subsection{Contact pressure}

The mean contact pressure (contact force divided by contact area) decreased when polyethylene thickness increased. On the articular surface of the polyethylene, the pattern of contact pressure followed an up and down movement during abduction (Fig. 3). The mean contact pressure was maximal at about $60^{\circ}$ of abduction. It reached 15.9, 12.6 and 11.6 MPa for 2, 4 and $6 \mathrm{~mm}$ of thickness. The contact pattern location was more inferior by nearly $1 \mathrm{~mm}$ as thickness increased by $2 \mathrm{~mm}$. This effect occurred mainly during the first $90^{\circ}$ of abduction. The peak contact pressure also decreased with polyethylene increase. It was maximal at $60^{\circ}$ of abduction. Local peak contact pressure reached 34.0, 23.9 and 20.4 MPa for 2, 4 and $6 \mathrm{~mm}$ of thickness. The

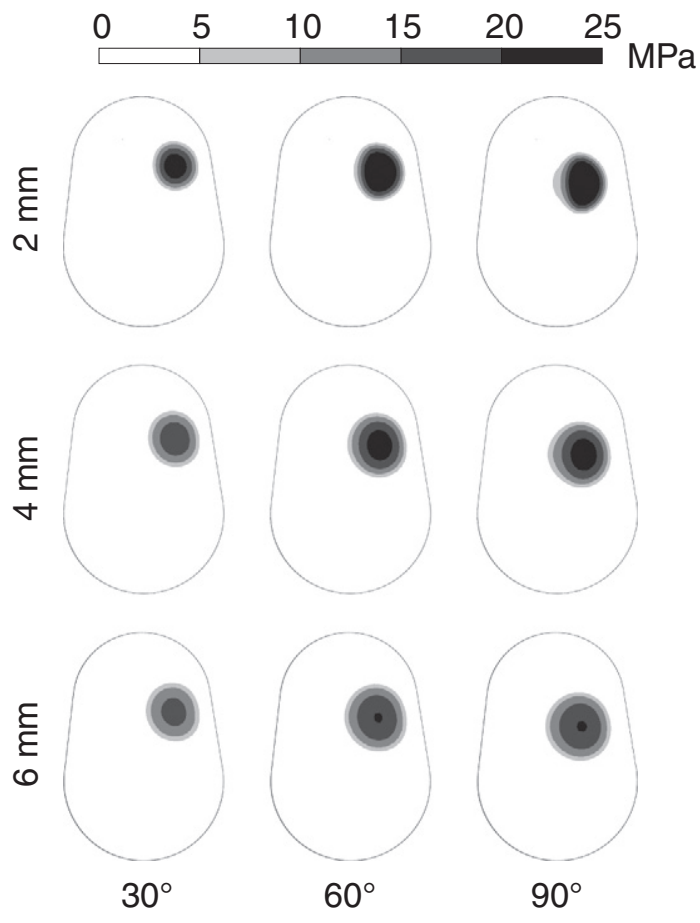

Fig. 3. Contact pressure on the glenoid component, for 2,4 and $6 \mathrm{~mm}$ of polyethylene thickness, at 30,60 and $90^{\circ}$ of abduction. decrease of contact pressure was more important between 2 and $4 \mathrm{~mm}$ than between 4 and $6 \mathrm{~mm}$.

\subsection{Polyethylene stress}

Within the polyethylene, the maximal value of the stress decreased as polyethylene thickness increased. It was maximal at $60^{\circ}$ of abduction. At this angle, the maximal stress was $28.3,21.2$ and $14.0 \mathrm{MPa}$ for 2,4 , and $6 \mathrm{~mm}$ of thickness. The maximal stress was nearly inversely proportional to polyethylene thickness $(-3.5 \mathrm{MPa} / \mathrm{mm})$. The volume of polyethylene with a stress above $20 \mathrm{MPa}$ was 16, 3, and $0 \mathrm{~mm}^{3}$ for 2, 4 and $6 \mathrm{~mm}$ of thickness (Fig. 4). The volume of higher stress was found between the articular contact point and opposite face at the backside of the glenoid plate.

\subsection{Cement stress}

Within the cement mantel, the stress decreased as the polyethylene thickness increased. It was maximal at $60^{\circ}$ of abduction. The maximal stress was 14.7, 9.0 and 7.8 MPa at 2, 4, and $6 \mathrm{~mm}$ of thickness. The decrease of stress was more important between 2 and $4 \mathrm{~mm}$ than between 4 and $6 \mathrm{~mm}$. The volume of cement with a stress above $5 \mathrm{MPa}$ was 107,68 , and $11 \mathrm{~mm}^{3}$ for 2, 4 and $6 \mathrm{~mm}$ of thickness (Fig. 5). The volume of higher (tensile) stress was mainly located around the contact point, below the posterior backside of the glenoid plate.

\subsection{Bone strain}

The volume of bone with a strain above $1 \%$ was 631,539 , and $294 \mathrm{~mm}^{3}$ for 2, 4, $6 \mathrm{~mm}$ respectively (Fig. 6).

\subsection{Interfacial shear stress}

At the implant-cement interface, the fraction of the interface (surface) with a shear stress above $5 \mathrm{MPa}$ (approximate failure limit) was $2.4,2.8$ and $0.5 \%$, for $2,4,6 \mathrm{~mm}$ of polyethylene thickness respectively. At the cement-bone interface, the fraction of the interface (surface) with a shear stress above $5 \mathrm{MPa}$ (approximate failure limit) was $4.0,2.0$ and $0.4 \%$, for $2,4,6 \mathrm{~mm}$ of polyethylene thickness respectively.

\section{Discusion}

The optimal thickness of the polyethylene glenoid component in anatomical total shoulder arthroplasty is still an open question. Reducing this thickness could be desired to improve the anatomical reconstruction of the articular surface, avoiding a stiffening of the surrounding soft tissue, but it might also overstress the polyethylene and the surrounding cement mantel. Using a numerical musculoskeletal model of the shoulder, we have evaluated the effect of the thickness of the polyethylene on the joint kinematics and mechanical stress within the polyethylene and cement. The model predicted a slight change in kinematics, but a large increase of stress within the polyethylene and the cement, particularly when the thickness was reduced by $2 \mathrm{~mm}$ from its reference value of $4 \mathrm{~mm}$. In addition, there was also an important drop of stress when the thickness was increased to $6 \mathrm{~mm}$, without any apparent drawback. Overall bone strain and interfacial shear stress were below their failure limits.

Our results suggest a weak potential advantage of using a thinner glenoid component to better reproduce the anatomy of the original articular surfaces. There was indeed nearly no change in kinematics or muscular moment arms between 2 and $6 \mathrm{~mm}$ of thickness. The global decrease of muscle moment arms as polyethylene thickness increase was caused by the associated lateralisation of the glenoid component and the rotation centre of the joint. The effect on the muscular 

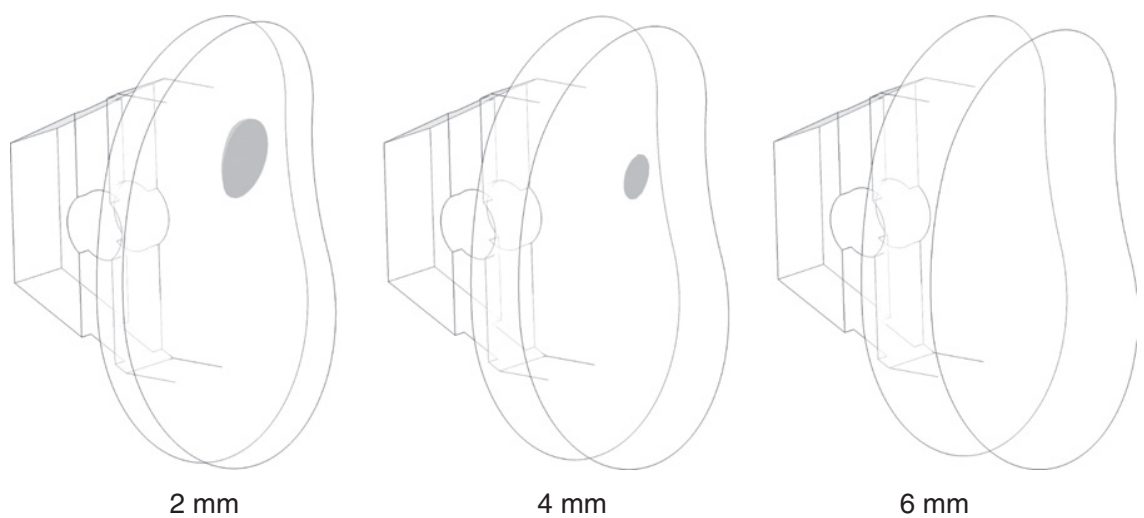

Fig. 4. Volume (grey zones) of the polyethylene component where the stress exceeds $20 \mathrm{MPa}$, for 2,4 and 6 mm of polyethylene thickness, at $60^{\circ}$ of abduction.

and contact forces was also rather weak. Even if it was not calculated explicitly, we assume that the effect on the stiffening of the soft tissue would be negligible (Strauss et al., 2009). On the other hand, we predicted a high increase of the stress state, at the articular surface, within the polyethylene and within the cement, when the thickness of the polyethylene was reduced to $2 \mathrm{~mm}$. The stress increase was particularly critical within the cement mantel. The bone strain was calculated, but not presented here since it was in overall below the approximate failure limit (1\%).

For the polyethylene, a yield stress value of about $20 \mathrm{MPa}$ is usually used as failure criterion (Swieszkowski et al., 2003), although its fatigue limit has been reported between 12 and $31 \mathrm{MPa}$ (Medel et al., 2007; Sauer et al., 1996). We have thus considered 20 MPa to evaluate the volume of polyethylene above these values. Note that there was no stress above $30 \mathrm{MPa}$ in our results. Peak stress was inversely proportional to the thickness, and producing a drop of the volume of polyethylene above the usual critical stress value of $20 \mathrm{MPa}$. Regarding the cement mantel, we considered a fatigue value of $5 \mathrm{MPa}$. This value was already estimated (Lacroix and Prendergast, 1997) from experimental measurement (Davies et al., 1987). Our model predicted an important volume of cement exceeding this fatigue limit, for $2 \mathrm{~mm}$ and $4 \mathrm{~mm}$ of thickness. This volume was mainly found behind the posterior side of the glenoid plate. With $6 \mathrm{~mm}$ of thickness, the volume of cement above the fatigue failure limit nearly vanished. The values of contact stress at the surface of the polyethylene and the von Mises stress within the polyethylene are of course related to damage and wear of the polyethylene, which is a common issue in total shoulder arthroplasty. The value of maximal principal (tensile) stress within the cement is associated to the micro and macroscopic fractures of the cement mantel. During this mechanical degradation, both materials can produce particles debris, which can eventually lead to implant loosening through inflammatory processes.

The reported values of humerus translation, muscle moment arms, and joint forces are consistent with the literature (Graichen et al., 2000; Hughes et al., 1998; Kelkar et al., 2001; Liu et al., 1997; Massimini et al., 2008; Poppen and Walker, 1978; Westerhoff et al., 2009). Using a 2D (axisymmetric) model of the glenoid component, Swieszkowski et al. also reported that increasing glenoid thickness reduces the maximal contact pressure (Swieszkowski et al., 2003). With a compression force of $1000 \mathrm{~N}$ of the humeral head, and the same radial mismatch as in our study $(6 \mathrm{~mm})$, they predicted a peak contact pressure of 35 and $26 \mathrm{MPa}$ with a respective thickness of 4 and $7 \mathrm{~mm}$. This 2D study compares rather well with our predictions, at least qualitatively. In a 3D numerical study, the effect of the thickness was evaluated for 2, 6 and $10 \mathrm{~mm}$ (Hopkins et al., 2007a, 2007b). This study reported a nearly constant peak contact pressure of about $8 \mathrm{MPa}$. With rather different mechanical conditions (nearly half contact force and double polyethylene elastic modulus), the order of magnitude was consistent with our study. The trend was however contrary to our results. The same study reported that the peak tensile stress within the cement decreased from 7.7 to $2.8 \mathrm{MPa}$ when the polyethylene thickness increased from 2 to $10 \mathrm{~mm}$. Here, the absolute values and trends are more or less the same, accounting for the difference of force amplitude. In our study, we evaluated the effect of the polyethylene thickness on the polyethylene stress, but we can also extrapolate on the polyethylene wear and damage, which is actually the real problem (Scarlat and Matsen, 2001). For total shoulder arthroplasty, a recent experimental study on a mechanical simulator reported a slight increase of polyethylene wear as polyethylene thickness increased from 4 to $7 \mathrm{~mm}$ (Swieszkowski et al., 2011). For total

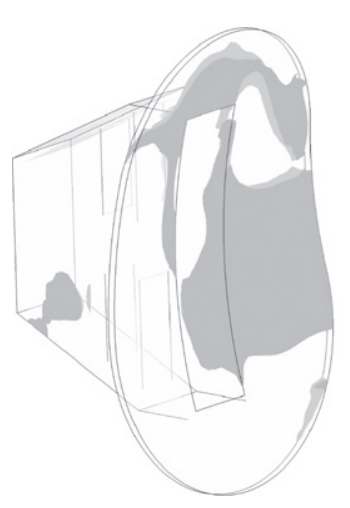

$2 \mathrm{~mm}$

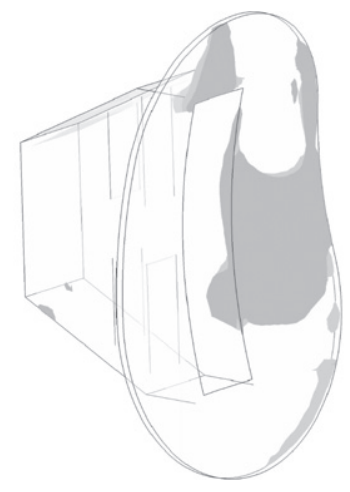

$4 \mathrm{~mm}$

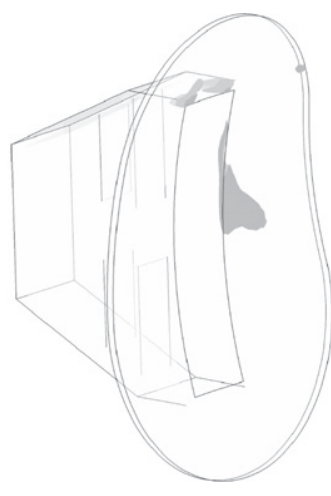

$6 \mathrm{~mm}$

Fig. 5. Volume (grey zones) of the cement layer where the stress exceeds $5 \mathrm{MPa}$, for 2,4 and $6 \mathrm{~mm}$ of polyethylene thickness, at $60^{\circ}$ of abduction. 


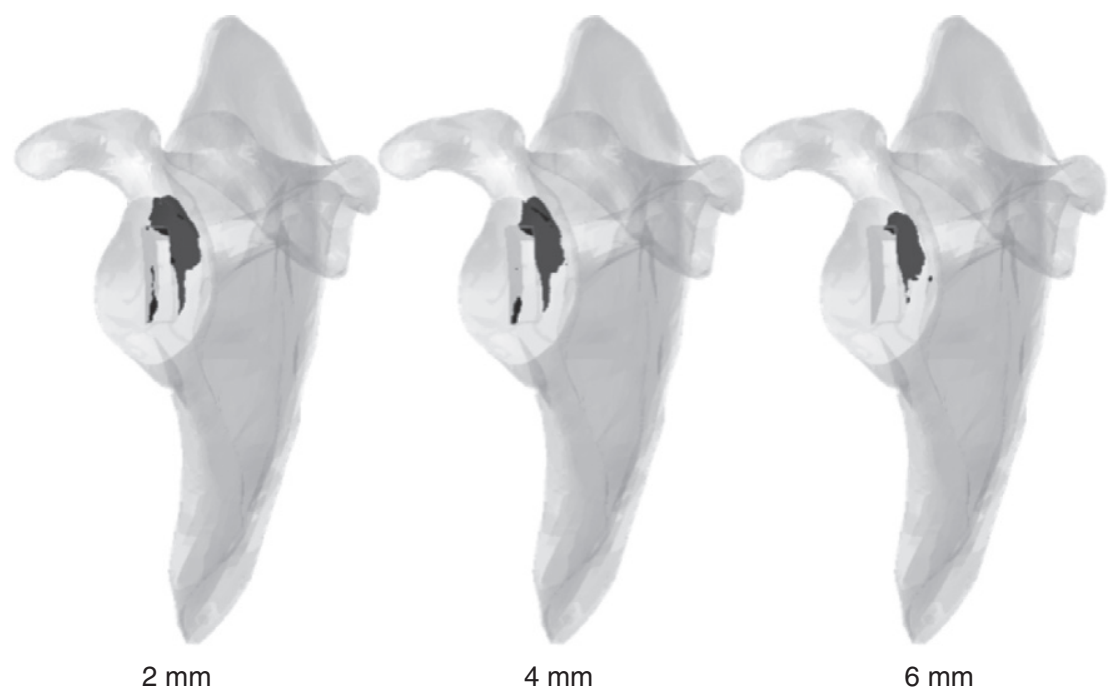

Fig. 6. Volume (grey zones) of the bone where the strain exceeds $1 \%$, for 2,4 and $6 \mathrm{~mm}$ of polyethylene thickness, at $60^{\circ}$ of abduction.

hip replacement, reducing the thickness of the polyethylene acetabular cup has been associated to an increase of wear for retrieved implants (Jasty et al., 1997; Oonishi et al., 1998). For the knee, a minimum thickness of $8 \mathrm{~mm}$ has been suggested (Bartel et al., 1986). Regarding the cement stress, there are no other studies reporting specifically the effect of the polyethylene thickness, but our results are in the same range of other numerical predictions of stress within the cement surrounding the glenoid component (Hopkins et al., 2004; Lacroix et al., 2000; Mansat et al., 2007). As for the knee, metal backing has been proposed to improve the fixation of the glenoid component. Although preliminary results were not satisfactory (Boileau et al., 2002), new design is still considered as an alternative to the all-polyethylene solution (Fucentese et al., 2010).

Numerical tools have been used widely to perform parametric analyses in total shoulder arthroplasty (Friedman et al., 1992; Lacroix and Prendergast, 1997; Lacroix et al., 2000; Mansat et al., 2007; Murphy et al., 2001; Orr et al., 1988; Stone et al., 1999). The present study is the first that focuses on the effect of the thickness of allpolyethylene components used in anatomical total shoulder arthroplasty. The originality of the numerical model used here is to use muscles both as motors and stabilisers of the joint. By this way, changes of prosthesis design are automatically associated with changes in joint kinematics, moment arms, muscle and joint forces. In addition, the model also accounts for the non-homogeneous distribution of the bone strength behind the glenoid component. These two features were essential to estimate the transmission of the muscular forces through the polyethylene, the cement and the glenoid bone. Failure of the polyethylene and cement is a complex phenomena, which is here roughly evaluated by a volume above a critical value (Davies et al., 1987; Kurtz et al., 1998; Pruitt, 2005), which can be associated to the fatigue stress, or endurance limit (Sauer et al., 1996). Soft tissue stiffening was not included in this study. We can obviously assume that every millimetre of polyethylene thickness above the pre-existing articular surface will extend the surrounding soft tissues by at most the same amount. The cement was represented by a uniform layer of constant thickness, which is often not the case for osteoporotic bone (Nyffeler et al., 2006). The stress within bone, cement and implant would of course depend on this thickness, as it has been observed previously (Terrier et al., 2005).

\section{Conclusions}

To conclude, this study confirms the hypothesis that the thickness of the polyethylene glenoid component can be related to glenoid loosening. More precisely, our results suggest that reducing the size of the glenoid component below $4 \mathrm{~mm}$ is not a reasonable option. Conversely, glenoid component with a thickness of $6 \mathrm{~mm}$ could be an interesting option, if the soft tissue tension allows it.

\section{Conflict of interest}

None.

\section{Acknowledgements}

This study was supported by the Center of Translational Biomechanics EPFL-CHUV-DAL and by Tornier (Tornier, Inc., Edina, MN, USA). The authors especially thank Yves-Alain Ratron (Research Director, Tornier) for his help, and Patricia Scheuber for the figures.

\section{References}

Bartel, D.L., Bicknell, V.L., et al., 1986. The effect of conformity, thickness, and material on stresses in ultra-high molecular weight components for total joint replacement. J. Bone Joint Surg. Am. 68 (7), 1041-1051.

Bohsali, K.I., Wirth, M.A., et al., 2006. Complications of total shoulder arthroplasty. J. Bone Joint Surg. Am. 88 (10), 2279-2292.

Boileau, P., Avidor, C., et al., 2002. Cemented polyethylene versus uncemented metalbacked glenoid components in total shoulder arthroplasty: a prospective, double-blind, randomized study. J. Shoulder Elbow Surg. 11 (4), 351-359.

Carter, D.R., Hayes, W.C., 1977. The compressive behavior of bone as a two-phase porous structure. J. Bone Joint Surg. Am. 59 (7), 954-962.

Davies, J.P., Burke, D.W., et al., 1987. Comparison of the fatigue characteristics of centrifuged and uncentrifuged Simplex P bone cement. J. Orthop. Res. 5 (3), 366-371.

Friedman, R.J., LaBerge, M., et al., 1992. Finite element modeling of the glenoid component: effect of design parameters on stress distribution. J. Shoulder Elbow Surg. 1, 261-270.

Fucentese, S.F., Costouros, J.G., et al., 2010. Total shoulder arthroplasty with an uncemented soft-metal-backed glenoid component. J. Shoulder Elbow Surg. 19 (4), 624-631.

Graichen, H., Stammberger, T., et al., 2000. Glenohumeral translation during active and passive elevation of the shoulder - a 3D open-MRI study. J. Biomech. 33 (5), 609-613.

Harryman, D.T., Sidles, J.A., et al., 1995. The effect of articular conformity and the size of the humeral head component on laxity and motion after glenohumeral arthroplasty. A study in cadavera. J. Bone Joint Surg. Am. 77 (4), 555-563.

Hopkins, A.R., Hansen, U.N., et al., 2004. The effects of glenoid component alignment variations on cement mantle stresses in total shoulder arthroplasty. J. Shoulder Elbow Surg. 13 (6), 668-675.

Hopkins, A.R., Hansen, U.N., et al., 2007a. Wear in the prosthetic shoulder: association with design parameters. J. Biomech. Eng. 129 (2), 223-230.

Hopkins, A.R., Hansen, U.N., et al., 2007b. Glenohumeral kinematics following total shoulder arthroplasty: a finite element investigation. J. Orthop. Res. 25 (1), 108-115.

Hughes, R.E., Niebur, G., et al., 1998. Comparison of two methods for computing abduction moment arms of the rotator cuff. J. Biomech. 31 (2), 157-160. 
Jasty, M., Goetz, D.D., et al., 1997. Wear of polyethylene acetabular components in total hip arthroplasty. An analysis of one hundred and twenty-eight components retrieved at autopsy or revision operations. J. Bone Joint Surg. Am. 79 (3), 349-358.

Kelkar, R., Wang, V.M., et al., 2001. Glenohumeral mechanics: a study of articular geometry, contact, and kinematics. J. Shoulder Elbow Surg. 10 (1), 73-84.

Kronberg, M., Nemeth, G., et al., 1990. Muscle activity and coordination in the normal shoulder. An electromyographic study. Clin. Orthop. Relat. Res. 257, 76-85.

Kurtz, S.M., Pruitt, L., et al., 1998. The yielding, plastic flow, and fracture behavior of ultra-high molecular weight polyethylene used in total joint replacements. Biomaterials 19 (21), 1989-2003.

Lacroix, D., Prendergast, P.J., 1997. Stress analysis of glenoid component designs for shoulder arthroplasty. Proc. Inst. Mech. Eng. H 211 (6), 467-474.

Lacroix, D., Murphy, L.A., et al., 2000. Three-dimensional finite element analysis of glenoid replacement prostheses: a comparison of keeled and pegged anchorage systems. J. Biomech. Eng. 122 (4), 430-436.

Liu, J., Hughes, R.E., et al., 1997. Roles of deltoid and rotator cuff muscles in shoulder elevation. Clin. Biomech. 12 (1), 32-38.

Mansat, P., Barea, C., et al., 1998. Anatomic variation of the mechanical properties of the glenoid. J. Shoulder Elbow Surg. 7 (2), 109-115.

Mansat, P., Briot, J., et al., 2007. Evaluation of the glenoid implant survival using a biomechanical finite element analysis: influence of the implant design, bone properties, and loading location. J. Shoulder. Elbow Surg. 16 (3S), S79-S83.

Massimini, D.F., Li, G., et al., 2008. Glenohumeral Articular Contact Kinematics of Patients after Total Shoulder Arthroplasty. 54th Annual Meeting of the Orthopaedic Research Society, San Francisco.

Medel, F.J., Pena, P., et al., 2007. Comparative fatigue behavior and toughness of remelted and annealed highly crosslinked polyethylenes. J. Biomed. Mater. Res. B Appl. Biomater. 83 (2), 380-390.

Murphy, L.A., Prendergast, P.J., et al., 2001. Structural analysis of an offset-keel design glenoid component compared with a center-keel design. J. Shoulder Elbow Surg. 10 (6), 568-579.

Nyffeler, R.W., Meyer, D., et al., 2006. The effect of cementing technique on structural fixation of pegged glenoid components in total shoulder arthroplasty. J. Shoulder Elbow Surg. 15 (1), 106-111.

Oonishi, H., Iwaki, H., et al., 1998. The effects of polyethylene cup thickness on wear of total hip prostheses. J. Mater. Sci. Mater. Med. 9 (8), 475-478.

Orr, T.E., Carter, D.R., et al., 1988. Stress analyses of glenoid component designs. Clin. Orthop. 232, 217-224.

Poppen, N.K., Walker, P.S., 1978. Forces at the glenohumeral joint in abduction. Clin. Orthop. 135, 165-170.
Pruitt, L.A., 2005. Deformation, yielding, fracture and fatigue behavior of conventiona and highly cross-linked ultra high molecular weight polyethylene. Biomaterials 26 (8), 905-915.

Rho, J.Y., Hobatho, M.C., et al., 1995. Relations of mechanical properties to density and CT numbers in human bone. Med. Eng. Phys. 17 (5), 347-355.

Rockwood, C.A., 2009. The Shoulder. PA, Saunders/Elsevier, Philadelphia.

Sauer, W.L., Weaver, K.D., et al., 1996. Fatigue performance of ultra-high-molecularweight polyethylene: effect of gamma radiation sterilization. Biomaterials 17 (20), 1929-1935.

Scarlat, M.M., Matsen III, F.A., 2001. Observations on retrieved polyethylene glenoid components. J. Arthroplasty 16 (6), 795-801.

Stone, K.D., Grabowski, J.J., et al., 1999. Stress analyses of glenoid components in total shoulder arthroplasty. J. Shoulder Elbow Surg. 8 (2), 151-158.

Strauss, E.J., Roche, C., et al., 2009. The glenoid in shoulder arthroplasty. J. Shoulder Elbow Surg. 18 (5), 819-833.

Swieszkowski, W., Bednarz, P., et al., 2003. Contact stresses in the glenoid component in total shoulder arthroplasty. Proc. Inst. Mech. Eng H 21 (1), 49-57.

Swieszkowski, W., Bersee, H.E.N., et al., 2011. Effect of the design parameters on the in vitro wear performance of total shoulder arthroplasties. Mater. Sci. Eng. C Mater. Biol. Appl. 31 (2), 313-319.

Terrier, A., Buchler, P., et al., 2005. Bone-cement interface of the glenoid component: stress analysis for varying cement thickness. Clin. Biomech. 20 (7), 710-717.

Terrier, A., Reist, A., et al., 2007. Effect of supraspinatus deficiency on humerus translation and glenohumeral contact force during abduction. Clin. Biomech. 22 (6), 645-651.

Terrier, A., Reist, A., et al., 2008a. Simulated joint and muscle forces in reversed and anatomic shoulder prostheses. J. Bone Joint Surg. Br. 90 (6), 751-756.

Terrier, A., Vogel, A., et al., 2008b. An algorithm to allow humerus translation in the indeterminate problem of shoulder abduction. Med. Eng. Phys. 30 (6), 710-716.

Terrier, A., Merlini, F., et al., 2009a. Comparison of polyethylene wear in anatomical and reversed shoulder prostheses. J. Bone Joint Surg. Br. 91 (7), 977-982.

Terrier, A., Merlini, F., et al., 2009b. Total shoulder arthroplasty: downward inclination of the glenoid component to balance supraspinatus deficiency. J. Shoulder Elbow Surg. 18 (3), 360-365.

Terrier, A., Ramondetti, S., et al., 2010. Biomechanical consequences of humeral component malpositioning after anatomical total shoulder arthroplasty. J. Shoulder Elbow Surg. 19 (8), 1184-1190 et al..

Westerhoff, P., Graichen, F., et al., 2009. In vivo measurement of shoulder joint loads during activities of daily living. J. Biomech. 42 (12), 1840-1849. 